\title{
Effect of IL-7 on Th17 cell responses in a mouse model of neutrophilic asthma
}

\author{
XIAOBO ZHANG ${ }^{1 *}$, MIN ZHANG $^{2 *}$, MIN JIANG $^{1}$ and GUANGMIN NONG ${ }^{1}$ \\ ${ }^{1}$ Pediatric Department, First Affiliated Hospital of Guangxi Medical University; ${ }^{2}$ Pediatric Department, People's Hospital \\ of Guangxi Zhuang Autonomous Region, Nanning, Guangxi Zhuang Autonomous Region 530021, P.R. China
}

Received July 4, 2019; Accepted April 21, 2020

DOI: $10.3892 / \mathrm{mmr} .2020 .11191$

\begin{abstract}
Neutrophilic asthma (NA) is characterized by neutrophil-mediated inflammation and the presence of Th17 cells. However, the mechanisms underlying Th17 cell responses in NA remain unknown. The aim of the present study was to examine the effects of interleukin (IL)-7 on Th17 cell responses in NA. A NA mouse model was sensitized by airway delivery of ovalbumin (OVA) and lipopolysaccharide and challenged with $1 \%$ OVA aerosol from day 21 for 3 consecutive days. Airway resistance was then measured to assess airway hyper-responsiveness (AHR). Cells from bronchoalveolar lavage fluid (BALF) underwent Diff-Quick and hematoxylin and eosin staining for classification. The levels of IL-17 in the BALF were determined by ELISA. The effects of IL-7 administration and STAT5 inhibition on Th17 cells were also characterized in vitro using splenic $\mathrm{CD}^{+}{ }^{+} \mathrm{T}$ cells. Ki-67, Bcl-2 and activated caspase- 3 expression in differentiated Th17 cells were analyzed by flow cytometry. The mouse model of NA was characterized by increased AHR, elevated levels of IL-17, high neutrophil counts in BALF, accumulated inflammatory cells in the lung and Th17 cell responses. IL-7 promoted the expression of Ki-67 and Bcl-2 while reducing caspase-3 expression. STAT5 inhibitor treatment decreased the levels of $\mathrm{Ki}-67$ and $\mathrm{Bcl}-2$, and resulted in increased expression of caspase-3. These results suggested that the IL-7/JAK/STAT5 signaling pathway may be involved in Th17 cell responses in NA.
\end{abstract}

Correspondence to: Professor Guangmin Nong, Pediatric Department, First Affiliated Hospital of Guangxi Medical University, 6 Shuangyong Road, Nanning, Guangxi Zhuang Autonomous Region 530021, P.R. China

E-mail: ngmprof8525@126.com

${ }^{*}$ Contributed equally

Abbreviations: NA, neutrophilic asthma; AHR, airway hyperresponsiveness; BALF, bronchio-alveolar lavage fluid

Key words: interleukin-7, JAK/STAT, Th17 cell, NA

\section{Introduction}

Asthma is a common chronic airway inflammatory disease with two subtypes, eosinophilic asthma and non-eosinophilic asthma. Neutrophilic asthma (NA) accounts for more than one-half of non-eosinophilic asthma (1-3). Corticosteroid treatment is effective for most patients with mild and moderate asthma. However, patients with NA require a high dose of corticosteroids for symptom control (4). An in-depth understanding of the mechanisms underlying NA would provide insight into therapeutic options for this condition.

Our previous studies (Jiang et al, unpublished data) demonstrated that Th17 cells were dominant and promoted neutrophil-mediated airway inflammation through interleukin (IL)-17 in a mouse model of NA. The elevated IL-6 and transforming growth factor- $\beta$ levels in NA model mice bronchio-alveolar lavage fluid (BALF) were discovered to participate in the Th17-mediated response through regulating the expression levels of retinoic acid receptor-related orphan receptor- $\gamma \mathrm{t}(\mathrm{ROR} \gamma \mathrm{t})$ and suppressor of cytokine signaling 3 . In addition, the increase in Th17 cells and ROR $\gamma \mathrm{t}$ expression in the peripheral blood, as well as upregulated sputum IL-17 levels, in children with NA were also verified. Moreover, the expression levels of phosphorylated (p)-STAT5 and Bcl-2 in Th17 cells and IL-7 levels in BALF were increased (Jiang et al, unpublished data). However, the effect of IL-7 on Th17 cells in NA remains unclear. It is known that IL-7 plays a critical role in proliferation, survival and differentiation of $\mathrm{T}$ lymphocytes. Indeed, IL-7 activates the JAK/STAT signaling pathway, thereby promoting $\mathrm{T}$ cell survival by upregulating the expression of the anti-apoptotic protein Bcl-2 (5-8). Therefore, it was hypothesized that the IL-7/JAK/STAT5 signaling pathway might also be involved in Th17 cell responses in NA.

\section{Materials and methods}

Experimental animals. A total of 12 female C57BL/6 mice (age, 6-8 weeks; weight, 18-20 g) were purchased from Shanghai SLAC Laboratory Animal Co., Ltd. and randomly divided into two groups (n=6 in each group), NA group and normal control (NC) group. Mice were housed under specific pathogen-free conditions in separate cages at a relatively stable temperature of $20-24^{\circ} \mathrm{C}$ and a humidity of $55 \pm 10 \%$, with a 12 -h light/dark cycle and free access to food and water. All experimental 
animal protocols were approved by The Ethics Committee of The First Affiliated Hospital of Guangxi Medical University [2019 (KY-E-035)].

Mouse model of NA. The model used in the present study was established according to the protocol from Wilson et al (9). Mice were sensitized by airway delivery of $100 \mu \mathrm{g}$ ovalbumin (OVA; Grade II \& V; Sigma-Aldrich; Merck KGaA) and $0.1 \mu \mathrm{g}$ lipopolysaccharide (LPS; Sigma-Aldrich; Merck KGaA) in a total volume of $50 \mu \mathrm{l}$ PBS on days 0,6 and 13. The OVA + LPS mixture was instilled along the posterior oropharyngeal wall, and the mixed solution was inhaled into the airway, followed by a challenge with $1 \%$ OVA aerosol for $1 \mathrm{~h}$ from day 21 for 3 consecutive days. The NC group received PBS treatment instead of OVA + LPS for sensitization and challenge.

Measurement of airway hyper-responsiveness (AHR). Airway responses to aerosolized methacholine were measured using a lung function test instrument for mouse (FinePointe Resistance and Compliance; Data Sciences International; Harvard Bioscience, Inc.). Mice were anesthetized with $1 \%$ pentobarbital sodium $(50 \mathrm{mg} / \mathrm{kg}$ body weight) by intraperitoneal injection, and the trachea was cannulated with a needle, followed by mechanical ventilation. Airway resistance $\left(\mathrm{R} ; \mathrm{cmH}_{2} \mathrm{O} . \mathrm{s} / \mathrm{ml}\right)$ was measured after aerosolization of $10 \mu \mathrm{l}$ PBS and administration of increasing doses of aerosolized methacholine (3.125, 6.25, 12.5, 25 and $50 \mathrm{mg} / \mathrm{ml}$ in $10 \mu \mathrm{l}$; Sigma-Aldrich; Merck KGaA) sequentially. The results are presented as fold-increase of $\mathrm{R}\left(\mathrm{cmH}_{2} \mathrm{O} . \mathrm{s} / \mathrm{ml}\right)$ above the baseline and were calculated as follows: [R(response) - R(baseline)]/R(baseline).

Cell classification of BALF. Mice were sacrificed $24 \mathrm{~h}$ after the final aerosolization. Cervical dislocation was used for euthanasia and death was confirmed by the onset of rigor mortis, according to The National Institutes of Health Guide for the Care and Use of Laboratory Animals. The trachea was exposed, and a 22-gauge needle was used for endotracheal intubation. The lungs were subjected to broncho-alveolar lavage twice with $0.5 \mathrm{ml}$ PBS (recovery rate $\geq 80 \%$ ) and the total volume of BALF was $0.8 \mathrm{ml}$. Total and differential cell counts from BALF were determined by staining with Diff-Quick (Beijing Solarbio Science \& Technology Co., Ltd.) for $1 \mathrm{~min}$ at room temperature. BALF was centrifuged at $160 \mathrm{x} \mathrm{g}$ for $10 \mathrm{~min}$ at $4^{\circ} \mathrm{C}$ and the supernatants were stored at $-20^{\circ} \mathrm{C}$ for further experiments.

Histopathology. Lungs were fixed in $4 \%$ paraformaldehyde solution for $24 \mathrm{~h}$ at room temperature and subjected to gradient alcohol dehydration and paraffin-embedding, which were cut into $5-7-\mu \mathrm{m}$ thick sections. The sections were subsequently stained with hematoxylin at room temperature for 2-3 min and then with eosin at room temperature for 30-60 sec. An Olympus CX31 light microscope (Olympus Corporation) was used to evaluate the general inflammation and the airway morphology (magnification, x200).

ELISA. An ELISA kit (cat. no. ELM-IL17-1; RayBiotech Life) was used to measure the levels of IL-17 in the BALF, according to the manufacturer's protocol.
Isolation of mononuclear cells from mouse spleens. Spleens were homogenized and filtered on a $0.054-\mathrm{mm}$ diameter 300 -mesh metal screen. The resulting cell suspension was centrifuged at $135 \mathrm{x}$ g for $5 \mathrm{~min}$ at $4^{\circ} \mathrm{C}$. Red blood cell lysis buffer $(3 \mathrm{ml})$ (Beijing Solarbio Science \& Technology Co., Ltd.) was added to the cell pellet and rested for $5 \mathrm{~min}$ at room temperature after thorough mixing. Subsequently, the reaction was stopped, and the supernatant discarded after centrifugation at $135 \mathrm{x} \mathrm{g}$ for $5 \mathrm{~min}$ at $4^{\circ} \mathrm{C}$. The cells were washed twice with cold PBS and centrifuged at $135 \mathrm{x}$ g for $5 \mathrm{~min}$ at $4^{\circ} \mathrm{C}$, before adjusting the cell concentration to $1 \times 10^{8}$ cells $/ \mathrm{ml}$. Subsequently, $20 \mu 1$ cell suspension were mixed with an equal volume of $2 \%$ Trypan Blue, then visually examined to confirm cell viability (unstained cells per $\mathrm{ml} /$ total cells per $\mathrm{ml}$ ) of $>95 \%$, using an Olympus CX31 light microscope (Olympus Corporation; magnification, $\mathrm{x} 200$ ).

Immunomagnetic bead separation of $C D 4^{+} T$ cells from splenic mononuclear cells. $\mathrm{CD}^{+}{ }^{+} \mathrm{T}$ cells were purified using a magnetic separation kit (Dynal; Thermo Fisher Scientific, Inc.). Splenic mononuclear cells from mice were mixed with the supplied antibody $\left(20 \mu 1 / 10^{7}\right.$ cells $)$, followed by inactivated fetal bovine serum (Wisent, Inc.; $20 \mu \mathrm{l} / 10^{7}$ cells). The reaction was mixed thoroughly and incubated for $20 \mathrm{~min}$ at $4^{\circ} \mathrm{C}$. After centrifugation at $211 \mathrm{x} \mathrm{g}$ for $5 \mathrm{~min}$ at $4^{\circ} \mathrm{C}$, the cells were resuspended in cold PBS ( $800 \mu 1 / 10^{7}$ cells), followed by magnetic beads and incubation for $15 \mathrm{~min}$ at room temperature. The system was placed in the automated cell selector for 2 min to collect the supernatant containing purified $\mathrm{CD}^{+}$ $\mathrm{T}$ cells, then centrifuged at $211 \mathrm{x} \mathrm{g}$ for $8 \mathrm{~min}$ at $4^{\circ} \mathrm{C}$. The cells were resuspended at a density of $1.5 \times 10^{6}$ cells $/ \mathrm{ml}$, and $100 \mu 1$ of this solution was incubated with PerCP-Cy ${ }^{\mathrm{TM}}$ 5.5-labeled anti-mouse CD4 monoclonal antibody (BD Biosciences; cat. no. 550954) at room temperature for $20 \mathrm{~min}$. Finally, the cells were washed with PBS before fixation with $1 \%$ paraformaldehyde for 10-20 min at $4^{\circ} \mathrm{C}$. Cell purity was then assessed by flow cytometry (to determine whether purity was $>91 \%$ ).

Culture of mouse splenic $C D 4^{+} T$ cells. Anti-CD3 (BD Biosciences; cat. no. 561798) and anti-CD28 (BD Biosciences; cat. no. 562764) antibodies were coated in each well on day 1 , followed by addition of anti-IFN (BD Biosciences; cat. no. 551506) and anti-IL-4 (BD Biosciences; cat. no. 555090). Purified $\mathrm{CD}^{+} \mathrm{T}$ cells were seeded into a 24 -well plate at a density of $1.5 \times 10^{6}$ cell per well in a $1 \mathrm{ml}$ volume. Subsequently, $10 \mathrm{ng} / \mathrm{ml}$ IL-7 (PeproTech, Inc.) and $100 \mu \mathrm{M}$ STAT5 inhibitor (Merck KGaA) were added to the culture, and incubated for $72 \mathrm{~h}$. The following culture groups were obtained: Negative control (NC) group, anti-CD3 + anti-CD28 + DMSO; $\mathrm{NC}+$ IL-7 group, anti-CD3 + anti-CD28 + IL-7 + DMSO; NA group, anti-CD3 + anti-CD28 + DMSO; NA + IL-7 group, anti-CD3 + anti-CD28 + IL-7 + DMSO; NA + IL-7 + STAT5 inhibitor group:, anti-CD3 + anti-CD28 + IL-7 + STAT5 inhibitor. The cells in each group were detected by a flow cytometer (FACS Calibur; BD Biosciences) and analyzed using FlowJo 7.6.5 software (FlowJo LLC).

Flow cytometric analysis. Briefly, cells from the spleen were stimulated in a complete medium with $50 \mathrm{ng} / \mathrm{ml}$ phorbol myristate acetate and $1 \mu \mathrm{g} / \mathrm{ml}$ ionomycin (both from 

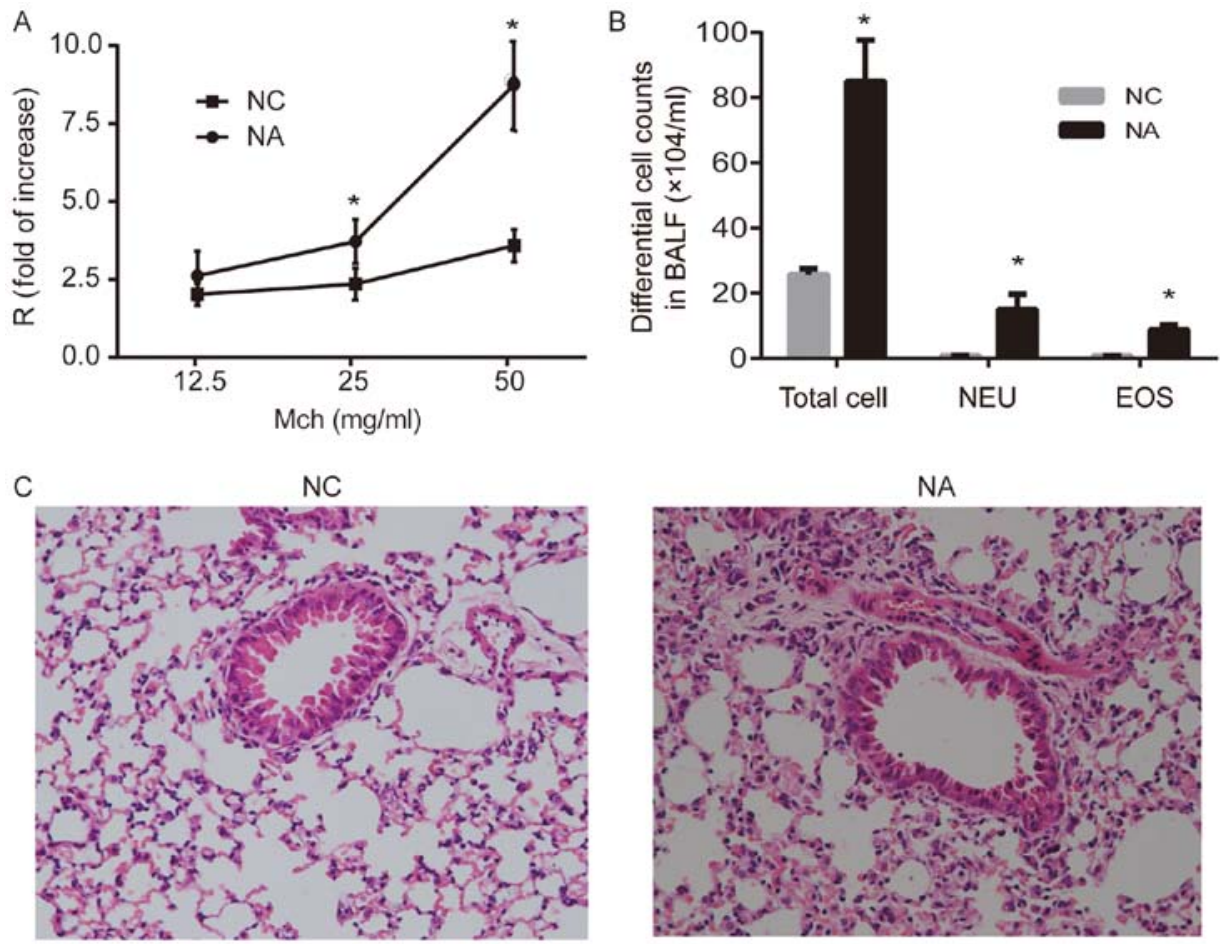

Figure 1. Airway resistance, differential cell counts and histopathology of the lung in a mouse model of NA. (A) Airway resistance increased significantly in NA. (B) Analysis of total and differential cell counts in BALF. Levels of NEU and EOS were increased in NA. (C) Representative images of hematoxylin and eosin-stained lung tissue. Disordered lung structure, widened alveolar septum, broken alveolar wall and infiltration of neutrophils around airways and in the interstitial pulmonary tissue was observed in NA (magnification, $x 100$ ). Data are presented as the mean \pm SD and analyzed by Student's $t$-test. $n=6$ in each group. " $\mathrm{P}<0.05$ vs. respective NC. NC, normal control; NA, neutrophilic asthma; Mch, methacholine; BALF, bronchoalveolar lavage fluid; NEU, neutrophils; EOS, eosinophils; $\mathrm{R}$, airway resistance.

Sigma-Aldrich; Merck $\mathrm{KGaA}$ ) at $37^{\circ} \mathrm{C}$ in $5 \% \mathrm{CO}_{2}$ for $5 \mathrm{~h}$. The cells were washed with PBS and stained with surface PerCP-Cy ${ }^{\mathrm{TM}} 5.5$ anti-mouse CD4 antibody (cat. no. 550954; clone, RM4-5; BD Biosciences) at room temperature in the dark for $30 \mathrm{~min}$. Intracellular staining for p-STAT5 and IL-17A was subsequently performed; briefly, the cells were fixed at $37^{\circ} \mathrm{C}$ for 10 min using warm BD Phosflow ${ }^{\mathrm{TM}}$ Fix Buffer I (cat. no. 557870; BD Biosciences) and washed with PBS. Subsequently, the cells were permeabilized for 30 min at $4^{\circ} \mathrm{C}$ using the BD Phosflow ${ }^{\mathrm{TM}}$ Perm Buffer III (cat. no. 558050; BD Biosciences), washed with PBS and stained with BD Phosflow ${ }^{\mathrm{TM}}$ Alexa Fluor ${ }^{\circledR} 488$ Anti-p-STAT5 (Y694; cat. no. 612598; clone, 47/Stat5 (pY694); BD Biosciences), BD Pharmingen $^{\mathrm{TM}}$ phycoerythrin (PE) anti-mouse IL-17A (cat. no. 559502; clone, TC11-18H1; BD Biosciences) or with its isotypic control antibody for $50 \mathrm{~min}$ at $4^{\circ} \mathrm{C}$.

Intracellular staining for IL-17A, active caspase-3, Ki-67 and $B c l-2$ was also performed. Briefly, the cells were fixed and permeabilized for $30 \mathrm{~min}$ at $4^{\circ} \mathrm{C}$ using the CytoFix/CytoPerm kit (cat. no. 554714; BD Biosciences), washed with PBS and stained with PE anti-mouse IL-17A (cat. no. 559502; clone, TC11-18H1; BD Biosciences), BD Transduction Laboratories ${ }^{\mathrm{TM}}$ FITC mouse anti-Ki-67 (cat. no. 612472; clone, 35/Ki-67; BD Biosciences), BD Pharmingen ${ }^{\text {TM }}$ FITC rabbit anti-active caspase-3 (cat. no. 560901; clone, C92-605; BD Biosciences), BD Pharmingen ${ }^{\mathrm{TM}}$ FITC hamster anti-mouse Bcl-2 (cat. no. 556357; BD Biosciences) or with its isotypic control antibody for $50 \mathrm{~min}$ at $4^{\circ} \mathrm{C}$. Live lymphocytes were gated according to forward and side scatter, then by CD4 expression. BD Pharmingen ${ }^{\mathrm{TM}}$ PE-Cy ${ }^{\mathrm{TM}} 5$ mouse IgG1 isotype control (cat. no. 550618; clone, MOPC-31C; BD Biosciences) was used as the isotype control. According to this gating strategy, $\mathrm{CD}^{+}$ cells were considered helper $\mathrm{T}(\mathrm{Th})$ cells; $\mathrm{CD} 4{ }^{+} \mathrm{IL}-17^{+}$cells were defined as Th17 cells. Ki-67, caspase- 3 and Bcl-2 expression in Th17 cells were measured as described. Cells were detected by a flow cytometer (FACS Calibur; BD Biosciences) and analyzed using FlowJo 7.6.5 software (FlowJo LLC).

Statistical analysis. Statistical analysis was performed using GraphPad Prism 6 software (GraphPad Software, Inc.). Normally distributed parameters are presented as the mean \pm SD of three independent experimental repeats. Statistical differences between two groups were determined by Student's t-test, and multi-group comparisons were carried out using one-way ANOVA after homogeneity test of variances. Tukey's multiple comparisons test was used after one-way ANOVA. $\mathrm{P}<0.05$ was considered to indicate a statistically significant difference.

\section{Results}

Establishment of a mouse model of NA. Airway resistance increased significantly in the NA group after challenge with 25 and $50 \mathrm{mg} / \mathrm{ml}$ methacholine, compared with the NC group $(\mathrm{P}<0.05$; Fig. 1A). The enumeration of total cells collected from BALF suggested that NA mice developed airway inflammation, and the numbers of neutrophils and eosinophils 

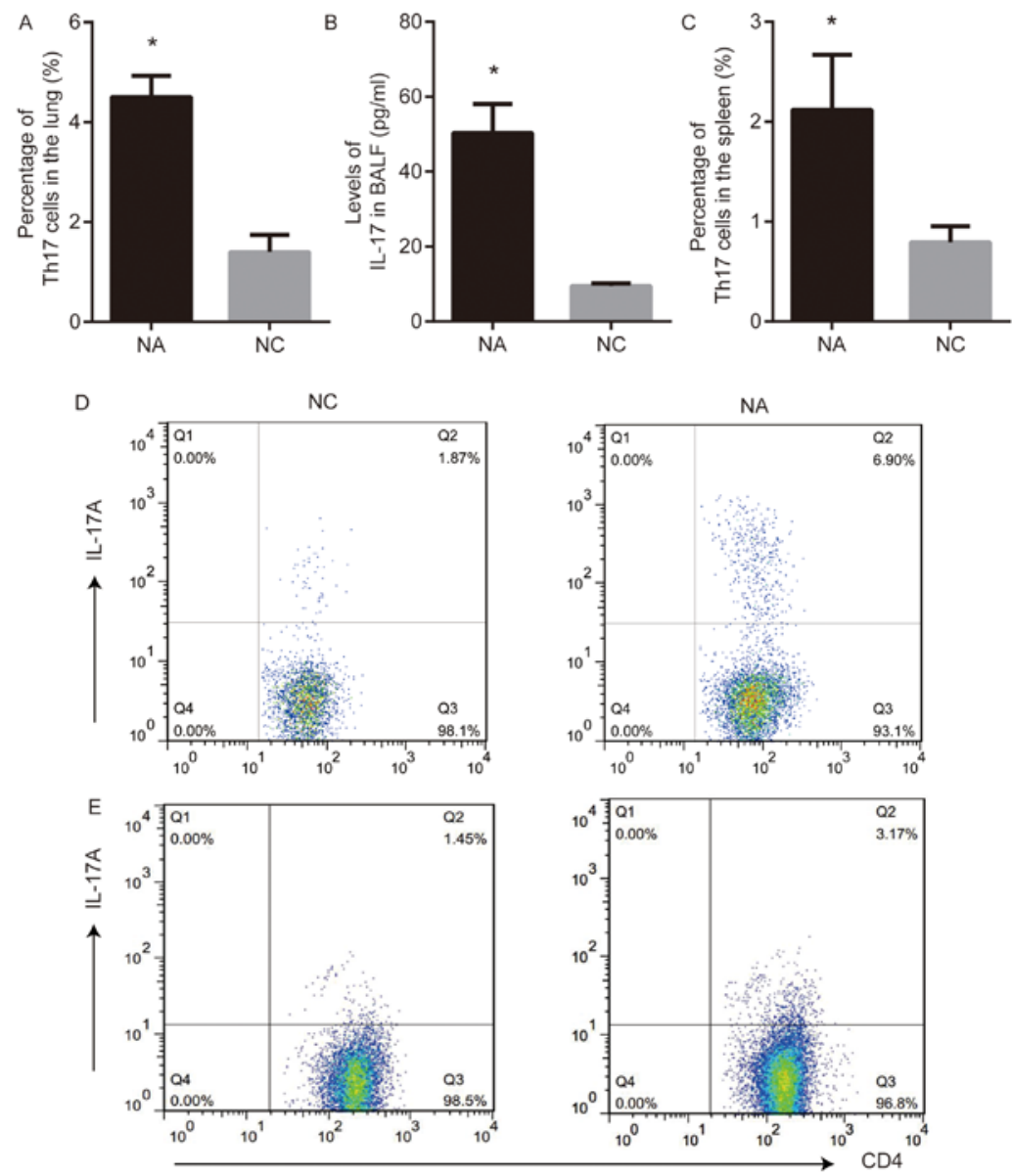

Figure 2. Th17 cell response is present in a mouse model of NA. (A) Percentage of Th17 cells increased in the lung of NA, as measured by flow cytometry. (B) Levels of IL-17 in BALF were elevated in NA, as measured by ELISA. (C) Expression of Th17 cells increased in the spleen of NA. (D) Flow cytometry demonstrated the increased frequency of Th17 cells in the lung of NA. (E) Flow cytometry demonstrated the increased percentage of Th17 cells in spleen from NA. Data are presented as the mean \pm SD and analyzed by Student's t-test. $n=6$ in each group. ${ }^{*} \mathrm{P}<0.05$ vs. NC. NC, normal control; NA, neutrophilic asthma; BALF, bronchoalveolar lavage fluid; IL, interleukin.

significantly increased in NA, compared with $\mathrm{NC}(\mathrm{P}<0.05$; Fig. 1B). Lung histopathology showed intact bronchial lumen and alveolar structure, aligned airway epithelial cells and no infiltration of the inflammatory cells in NC. However, disordered lung structure, widened alveolar septum, broken alveolar wall, and inflammatory cell infiltration (mainly neutrophils) were observed around airways and the interstitial pulmonary in NA (Fig. 1C).

Th17 cell expression in a mouse model of NA. Th17 cells and the levels of IL-17 were analyzed using flow cytometry and ELISA, respectively. Th17 cell frequency in the lung and the level of IL-17 in BALF from NA mice were higher than those in $\mathrm{NC}(\mathrm{P}<0.05$; Fig. 2A, B and D). The frequency of Th17 cells in the spleens from NA mice was higher than that in NC $(\mathrm{P}<0.05$; Fig. 2C and $\mathrm{E})$.

IL-7 affects the expression of proliferation markers and anti-apoptotic proteins in Th17 cells in NA mice. In the NC group, there were no differences in $\mathrm{Ki}-67$ and caspase-3 expression in the presence or absence of IL-7 (Fig. S1). IL-7 promoted the expression of the proliferation marker Ki-67 ( $\mathrm{P}<0.05$; Fig. 3A), anti-apoptotic protein Bcl-2 ( $<<0.05$; Fig. $3 \mathrm{~B})$ and p-STAT5 $(\mathrm{P}<0.05$; Fig. $3 \mathrm{C}$ and $\mathrm{D})$ in Th17 cells from NA mice, compared with untreated cells. However, IL-7 decreased activated caspase-3 expression in NA ( $\mathrm{P}<0.05$; Fig. 3A).

Expression of proliferating and anti-apoptotic proteins is affected by JAK/STAT pathway inhibition in Th17 cells from NA mice. Compared with NA mice treated with IL-7, STAT5 inhibition decreased the expression of Ki-67 (P<0.01; Fig. 4) and $\mathrm{Bcl}-2(\mathrm{P}<0.05$; Fig. 5) and increased the expression of caspase-3 $(\mathrm{P}<0.01$; Fig. 6$)$ in Th17 cells.

\section{Discussion}

NA is associated with neutrophil responses not only in severe asthmatics but also in patients with mild and moderate asthma (10). Our previous studies demonstrated that NA mice displayed increased levels of IL-7 and Th17 cell immune responses, as well as IL-17 secreted by Th17 cell-mediated neutrophilic airway inflammation (Jiang et al, unpublished data). However, the mechanisms underlying Th17 cell responses in NA remain unknown. Our previous study demonstrated that sputum supernatant from patients with NA inhibited apoptosis of peripheral blood neutrophils in normal individuals, which suggested a role for the airway micro-environment in the regulation of neutrophil airway inflammation (11). Several previous 
A

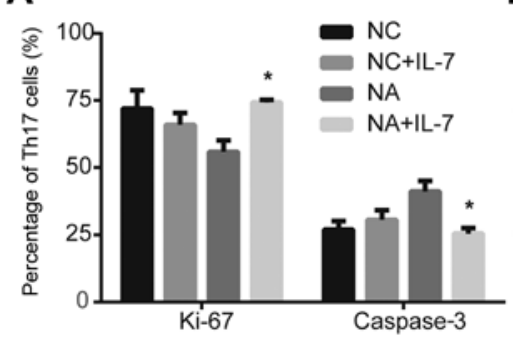

B

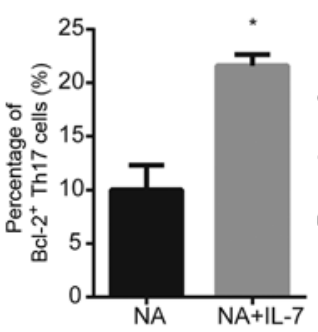

C

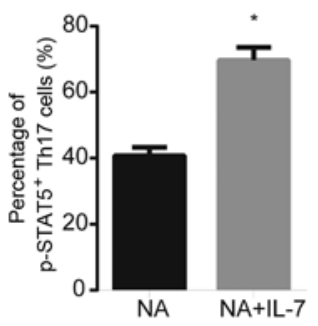

D

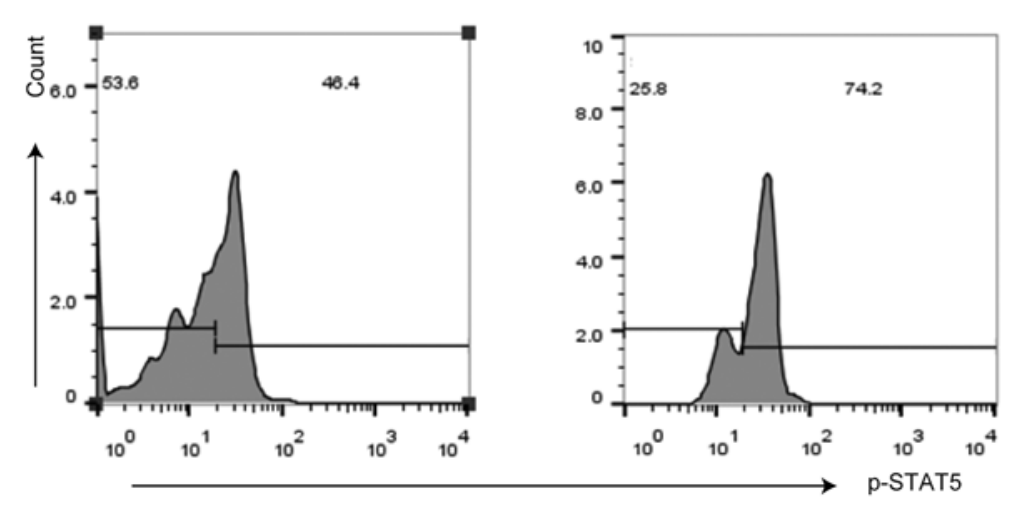

Figure 3. Expression of proliferation markers and anti-apoptotic proteins in Th17 cells from NA mice is affected by IL-7. (A) IL-7 promoted the expression of $\mathrm{Ki}-67$ and decreased caspase-3 expression in Th17 cells from NA mice. ${ }^{*} \mathrm{P}<0.05$ vs. respective NC. (B) IL-7 increased expression of anti-apoptotic protein Bcl-2 in Th17 cells of NA. (C and D) Expression of p-STAT5 was increased by IL-7. ${ }^{*}<0.05$ vs. NA. Data are presented as the mean \pm SD and analyzed by one-way ANOVA or Student's t-test. NC, normal control; NA, neutrophilic asthma; IL-7, interleukin-7; p, phosphorylated.

A

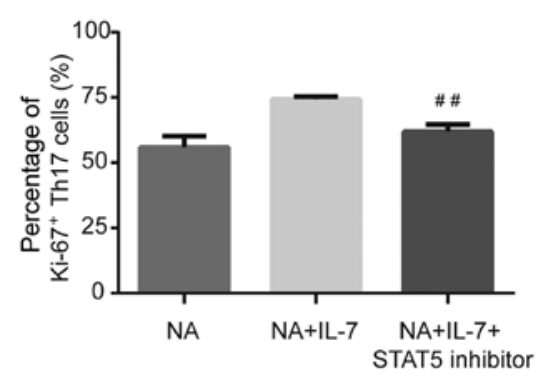

C

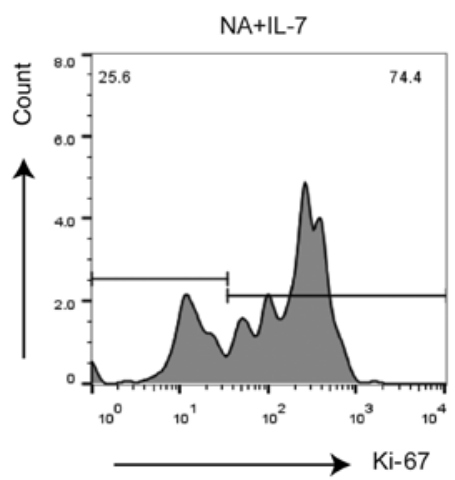

B

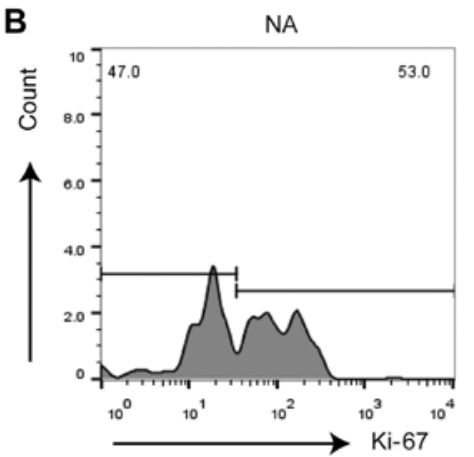

D

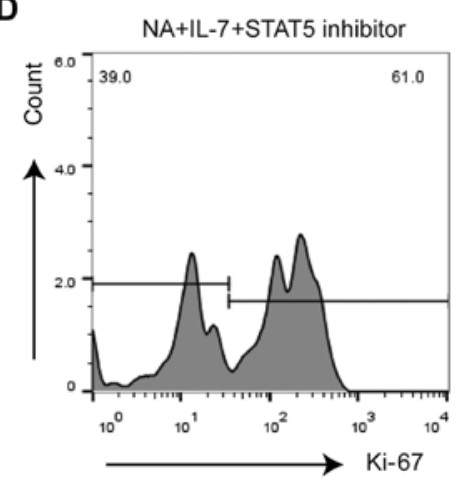

Figure 4. Expression of proliferation marker Ki-67 in Th17 cells from NA mice is altered by inhibition of the JAK/STAT pathway. (A) STAT5 inhibitor decreased the expression of Ki-67 in splenic Th17 cells from NA mice. (B) Expression of Ki-67 by flow cytometry in NA. (C) Ki-67 expression by flow cytometry in NA + IL-7. (D) Expression of Ki-67 by flow cytometry in the NA + IL-7 + STAT5 inhibitor group. ${ }^{\# / P}<0.01$ vs. NA + IL-7 group. NA, neutrophilic asthma; IL-7, interleukin-7.

studies demonstrated that IL-7 induced the expression of the anti-apoptotic protein Bcl-2 and activated the JAK/STAT signaling pathway, which contributed to T cell survival (6-8).
Therefore, it was hypothesized that IL-7 was involved in the Th17 responses seen in NA. In the present study, an NA mouse model was established, in which Th17 cells were affected by 

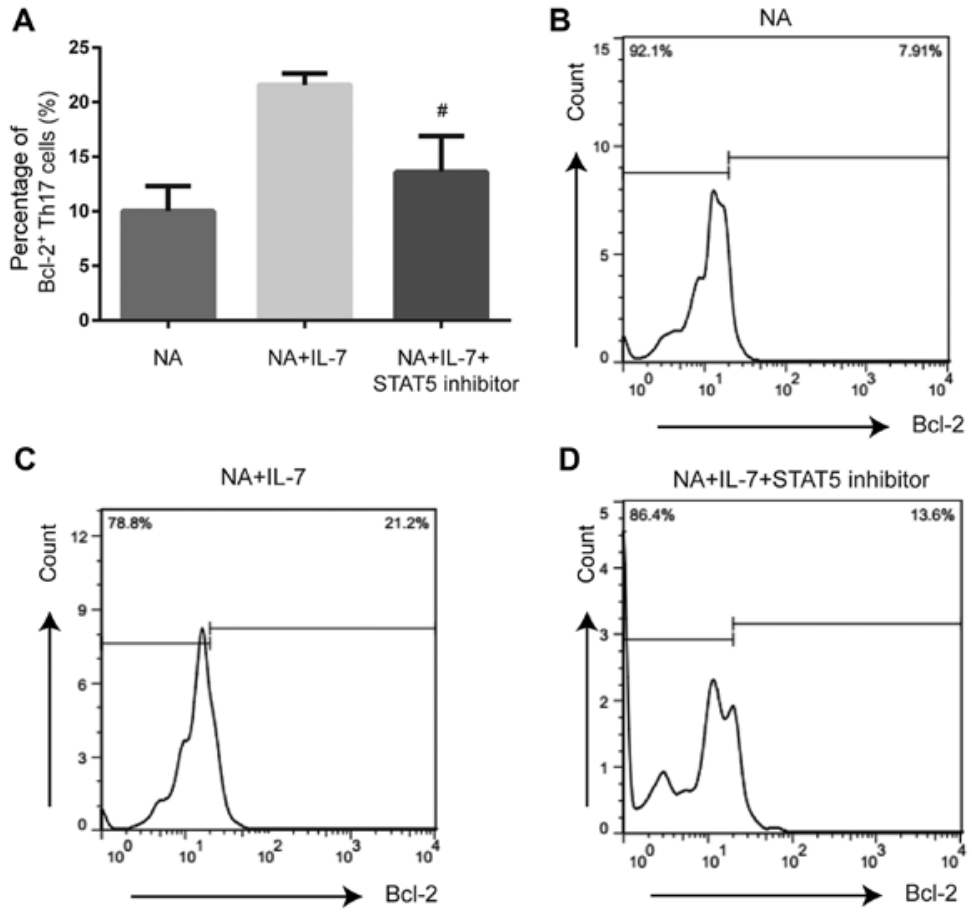

Figure 5. Expression of anti-apoptotic protein Bcl-2 in Th17 cells from NA mice is altered by inhibition of the JAK/STAT pathway. (A) STAT5 inhibitor reduced the expression of Bcl-2 in splenic Th17 cells from NA mice. (B) Expression of Bcl-2 in NA. (C) Bcl-2 expression by flow cytometry in NA + IL-7. (D) Expression of Bcl-2 in the NA + IL-7 + STAT5 inhibitor group. ${ }^{~} \mathrm{P}<0.05$ vs. NA + IL-7 group. NA, neutrophilic asthma; IL-7, interleukin-7.
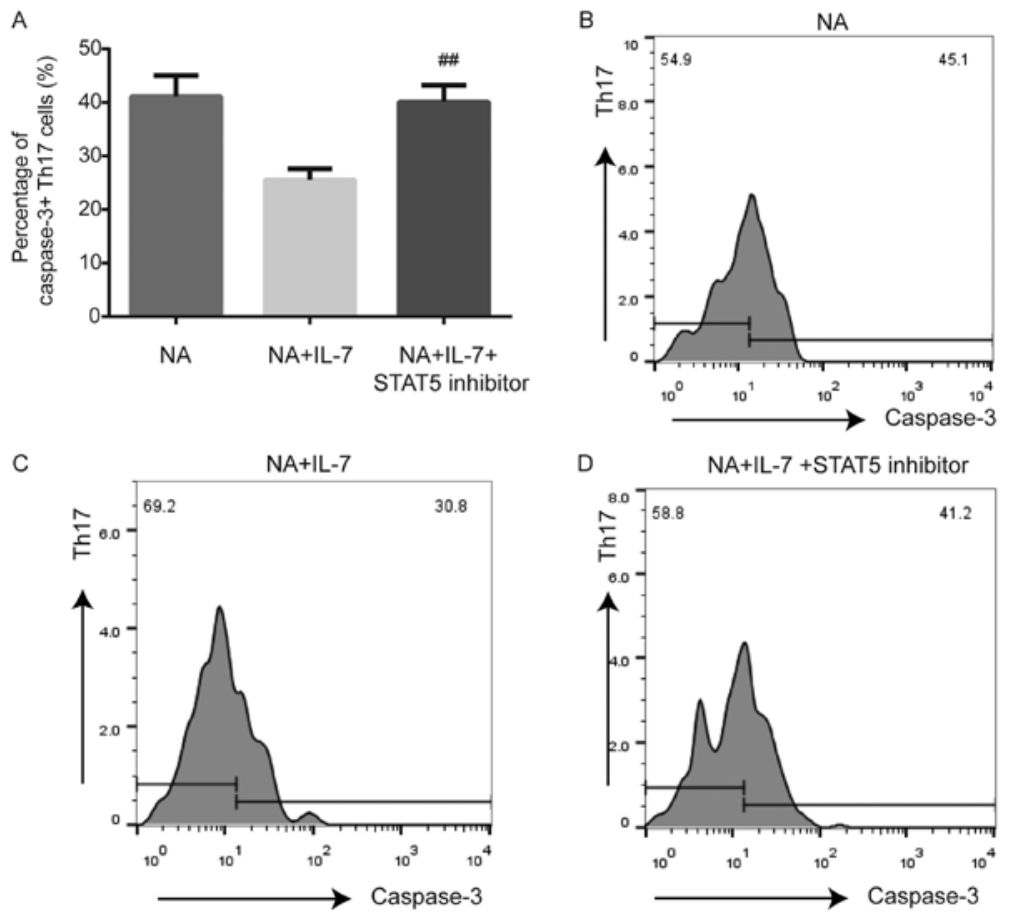

Figure 6. Caspase-3 expression in Th17 cells from NA mice is affected by inhibition of the JAK/STAT pathway. (A) STAT5 inhibitor promoted the expression of caspase-3 in Th17 cells of the spleen in NA mice. (B) Expression of caspase-3 by flow cytometry in NA. (C) Caspase-3 expression by flow cytometry in NA + IL-7. (D) Caspase-3 expression by flow cytometry in the NA + IL-7 + STAT5 inhibitor group. ${ }^{\# \# ~} \mathrm{P}<0.01$ vs. NA + IL-7 group. NA, neutrophilic asthma; IL-7, interleukin-7.

IL-7 and inhibited by suppression of the JAK/STAT pathway. Thus, the IL-7/JAK/STAT signaling pathway was involved in Th17 cell responses in this model of NA.

The results from a previous study on the conventional animal model of asthma sensitized by intraperitoneal injection were inconsistent with those of NA and did not reflect the exact state of airway inflammation and AHR (9). Thus, a mouse model of NA might provide insight into the mechanisms underlying these differences. A previous study demonstrated that allergic sensitization of the airway stimulated robust Th17 
responses, and that neutrophilia was required for AHR (9). In the present study, a mouse model of NA was successfully established by airway delivery of OVA and LPS, based on the previous study by Wilson et al (9). NA mice displayed increased AHR, elevated levels of IL-17 and a high number of neutrophils in BALF. Moreover, numerous inflammatory cells infiltrated around the bronchus and blood vessels. In summary, the current mouse model presented the following features of NA: i) Presence of AHR; ii) accumulation of inflammatory cells in the lung, primarily with increased neutrophils; and iii) high number of neutrophils in BALF.

Accumulating evidence suggests that Th17 cells are involved in the pathogenesis of asthma $(9,12)$, including severe forms of asthma that are refractory to treatment with corticosteroids (13). IL-17 production by Th17 cells is associated with asthma AHR (13), corticosteroid resistance, goblet cell hypersecretion, airway fibrosis and airway remodeling (14). In the present study, NA mice demonstrated strong Th17 responses in the lung and spleen. Moreover, Th17 cell frequency, the level of IL-17 and AHR increased in NA mice when compared with the NC, which was was consistent with a previous study, in which Th17 cells were associated with neutrophilic airway inflammation (15).

IL-7 promotes airway inflammation by activating and maintaining eosinophil survival (16). However, the effects of IL-7 on neutrophils remain unclear. IL-7 is a member of the type I cytokine receptor family and plays a critical role in proliferation, survival and differentiation of $\mathrm{T}$ lymphocytes $(6,17)$. Bcl-2 is a crucial anti-apoptotic protein (18), and IL-7 can upregulate Bcl-2 expression (19). Liu et al (20) demonstrated that IL-7 promoted the survival and inhibited Th17 cell apoptosis in auto-immune encephalomyelitis mice. $\mathrm{Ki}-67$ is a nuclear protein associated with cellular proliferation (21), and caspase-3 plays a key role in the execution phase of cell apoptosis (22). The present study was consistent with the findings of Liu et al (20), in which IL-7 administration in NA resulted in increased levels of $\mathrm{Ki}-67$ and $\mathrm{Bcl}-2$, suggesting IL-7 is likely involved in Th17 cell proliferation. Furthermore, lymphocytes treated with caspase-3 inhibitor showed reduced apoptosis (23); IL-7 inhibited caspase-3 activation and reduced $\mathrm{T}$ cell apoptosis (5). In the present study, IL-7 administration reduced caspase-3 expression, which suggested that IL-7 was involved in inhibiting Th17 cell apoptosis. These data demonstrated that IL-7 was involved in the response of Th17 cells.

The JAK/STAT signaling pathway is involved in proliferation, differentiation and survival of immune cells (24). Previous studies suggested that the JAK/STAT5 signaling pathway played a role in asthma pathogenesis, and that JAK inhibition significantly antagonized p-STAT5 activation in T cells (25). However, only one previous study suggested that JAK/STAT5 signaling was involved in NA airway inflammation (26). Moreover, the JAK/STAT signaling pathway activated by IL-7 might be the mechanism underlying $\mathrm{T}$ cell survival and the development of corticosteroid resistance in asthma (27). The present study demonstrated that IL-7 increased the expression of p-STAT5, and the downstream proteins $\mathrm{Ki}-67$ and $\mathrm{Bcl}-2$ in Th17 cells from NA mice. Moreover, STAT5 inhibition reversed the effect of IL-7 on Ki-67, Bcl-2 and caspase-3 expression in Th17 cells. Thus, IL-7/JAK/STAT5 played a role in the prevalence of Th17 cells in NA mice.
In conclusion, the present study successfully established an NA mouse model by airway delivery of OVA in the presence of LPS. Furthermore, the response of Th17 cells in this model was identified. IL-7 regulated the expression of the proliferation marker Ki-67, anti-apoptotic protein Bcl-2 and pro-apoptotic protein caspase-3, and STAT5 inhibition could reverse this effect. To the best of the authors' knowledge, this is the first study on the contribution of IL-7 to Th17 responses in NA. A limitation of the present study is that the mechanism has not yet been verified in vivo. When assessing the response to IL-7, the lack of a positive control lymphocyte population was another limitation of the present study. Thus, further studies in mice are essential to determine whether IL-7 and JAK/STAT5 pathway blockade are a potential therapeutic approach for NA.

\section{Acknowledgements}

Not applicable.

\section{Funding}

The present study was supported by International Communication of Guangxi Medical University Graduate Education, Guangxi Natural Science Foundation (grant no. 2018GXNSFAA281256), Innovation Project of Guangxi Graduate Education (grant no. YCBZ2019045), Chen Xiaoping Foundation for the Development of Science and Technology of Hubei Province (grant no. CXPJJH1 1900003-17), Guangxi Health Commission Project (grant no. Z20190762) and The Education Foundation of Guangxi (grant no. 2017KY0117).

\section{Availability of data and materials}

All data generated or analyzed during the current study are available from the corresponding author on reasonable request.

\section{Authors' contributions}

$\mathrm{XZ}$ performed the experiments and analyzed the data, as well as prepared the manuscript. $\mathrm{MZ}$ performed the experiments and interpreted the data, as well as drafted the paper. MJ designed the study and revised the manuscript for important intellectual content. GN contributed to the conception of this study and overall supervision. All authors read and approved the final version.

\section{Ethics approval and consent to participate}

The present study was approved by The Ethics Committee of The First Affiliated Hospital of Guangxi Medical University [approval no. 2019(KY-E-035)].

\section{Patient consent for publication}

Not applicable.

\section{Competing interests}

The authors declare that they have no competing interests. 


\section{References}

1. Hekking PP and Bel EH: Developing and emerging clinical asthma phenotypes. J Allergy Clin Immunol Pract 2: 671-80; quiz 81, 2014.

2. Simpson JL, Scott R, Boyle MJ, Gibson PG: Inflammatory subtypes in asthma: assessment and identification using induced sputum. Respirology 11: 54-61, 2006.

3. Tsoumakidou M, Papadopouli E, Tzanakis N, Siafakas NM: Airway inflammation and cellular stress in noneosinophilic atopic asthma. Chest 129: 1194-1202, 2006.

4. Basyigit I, Yildiz F, Ozkara SK, Boyaci H, Ilgazli A: Inhaled corticosteroid effects both eosinophilic and non-eosinophilic inflammation in asthmatic patients. Mediators Inflamm 13: 285-291, 2004.

5. Chetoui N, Boisvert M, Gendron S, Aoudjit F: Interleukin-7 promotes the survival of human $\mathrm{CD}^{+}$effector/memory $\mathrm{T}$ cells by up-regulating Bcl-2 proteins and activating the JAK/STAT signalling pathway. Immunology 130: 418-426, 2010.

6. Fry TJ and Mackall CL: The many faces of IL-7: from lymphopoiesis to peripheral $\mathrm{T}$ cell maintenance. J Immunol 174: 6571-6576, 2005.

7. Zaunders JJ, Levy Y and Seddiki N: Exploiting differential expression of the IL-7 receptor on memory T cells to modulate immune responses. Cytokine Growth Factor Rev 25: 391-401, 2014.

8. Read KA, Powell MD, McDonald PW and Oestreich KJ: IL-2, IL-7, and IL-15: Multistage regulators of CD4(+) T helper cell differentiation. Exp Hematol 44: 799-808, 2016.

9. Wilson RH, Whitehead GS, Nakano H, Free ME, Kolls JK and Cook DN: Allergic sensitization through the airway primes Th17-dependent neutrophilia and airway hyperresponsiveness. Am J Respir Crit Care Med 180: 720-730, 2009.

10. Gauthier M, Ray A and Wenzel SE: Evolving Concepts of Asthma. Am J Respir Crit Care Med 192: 660-668, 2015.

11. Uddin M, Nong G, Ward J, Seumois G, Prince LR, Wilson SJ, Cornelius V, Dent G, Djukanovic R: Prosurvival activity for airway neutrophils in severe asthma. Thorax 65: 684-689, 2010.

12. Choy DF, Hart KM, Borthwick LA, Shikotra A, Nagarkar DR, Siddiqui S, Jia G, Ohri CM, Doran E, et al: TH2 and TH17 inflammatory pathways are reciprocally regulated in asthma. Sci Transl Med 7: 301ra129, 2015.

13. Newcomb DC and Peebles RS Jr: Th17-mediated inflammation in asthma. Curr Opin Immunol 25: 755-760, 2013.

14. Cosmi L, Liotta F and Annunziato F: Th17 regulating lower airway disease. Curr Opin Allergy Clin Immunol 16: 1-6, 2016.
15. Kudo M, Melton AC, Chen C, Engler MB, Huang KE, Ren X, Wang Y, Bernstein X, Li JT, et al: IL-17A produced by alphabeta $T$ cells drives airway hyper-responsiveness in mice and enhances mouse and human airway smooth muscle contraction. Nat Med 18: 547-554, 2012.

16. Kelly EA, Koziol-White CJ, Clay KJ, Liu LY, Bates ME, Bertics PJ, Jarjour NN: Potential contribution of IL-7 to allergen-induced eosinophilic airway inflammation in asthma. J Immunol 182: 1404-1410, 2009.

17. Sportes C, Hakim FT, Memon SA, Zhang H, Chua KS, Brown MR, Fleisher TA, Krumlauf MC, Babb RR, et al: Administration of rhIL-7 in humans increases in vivo TCR repertoire diversity by preferential expansion of naive T cell subsets. J Exp Med 205: 1701-1714, 2008.

18. Kale J, Osterlund EJ, Andrews DW: BCL-2 family proteins: changing partners in the dance towards death. Cell Death Differ 25: 65-80, 2018.

19. Goodsell DS: The molecular perspective: Bcl-2 and apoptosis. Oncologist 7: 259-260, 2002.

20. Liu X, Leung S, Wang C, Tan Z, Wang J, Guo TB, Fang L, Zhao Y, Wan B, et al: Crucial role of interleukin-7 in Thelper type 17 survival and expansion in autoimmune disease. Nat Med 16: 191-197, 2010.

21. Sun X and Kaufman PD: Ki-67: more than a proliferation marker. Chromosoma 127: 175-186, 2018.

22. Burgon PG and Megeney LA: Caspase signaling, a conserved inductive cue for metazoan cell differentiation. Semin Cell Dev Biol 11: 01-09, 2017.

23. Hotchkiss RS, Coopersmith CM and Karl IE: Prevention of lymphocyte apoptosis - a potential treatment of sepsis? Clin Infect Dis 41 (Suppl 7): S465-S469, 2005.

24. Harrison DA: The Jak/STAT pathway. Cold Spring Harb Perspect Biol 4: a011205, 2012.

25. Howell MD, Fitzsimons C, Smith P: JAK/STAT inhibitors and other small molecule cytokine antagonists for the treatment of allergic disease. Ann Allergy Asthma Immunol 120: 367-375, 2018.

26. Li RF and Wang GF: JAK/STAT5 signaling pathway inhibitor ruxolitinib reduces airway inflammation of neutrophilic asthma in mice model. Eur Rev Med Pharmacol Sci 22: 835-843, 2018.

27. Liu S, Verma M, Michalec L, Liu W, Sripada A, Rollins D, Good J, Ito Y, Chu H, et al: Steroid resistance of airway type 2 innate lymphoid cells from patients with severe asthma: The role of thymic stromal lymphopoietin. J Allergy Clin Immunol 141: 257-68.e6, 2018.

(i) () This work is licensed under a Creative Commons c) AY NO Attribution-NonCommercial-NoDerivatives 4.0 International (CC BY-NC-ND 4.0) License. 